\title{
Rechnergestütztes Operieren an der Wirbelsäule
}

\author{
Paul Alfred Grützner, Bernd Vock, Thorsten Köhler, Andreas Wentzensen
}

\section{Zusammenfassung}

Computerassistierte Operationsverfahren in der Unfallchirurgie und Orthopädie finden seit ihrer Einführung eine zunehmende Verbreitung und sind an der Schwelle vom experimentell wissenschaftlichen Ansatz zur Routineanwendung. Die Faszination der Navigationssysteme liegt in einer Verknüpfung der Bildinformation, sei es aus Schnittbildverfahren oder aus konventionellen Bildwandlerdaten, mit der intraoperativen Instrumentenposition. In den konventionellen Techniken muss diese Verknüpfung der teilweise sehr komplexen Bilddaten mit der Position des Instrumentes im Kopf des Operateurs erfolgen. Ein Informationsverlust ist hierbei nahezu unvermeidbar. Als Bildgebung steht im Operationssaal in der Regel lediglich ein Röntgenbildverstärker, der eine Instrumentenposition in Echtzeit nur in einer Ebene verfolgen kann, zur Verfügung. Die in einzelnen Kliniken verfügbaren intraoperativen Möglichkeiten einer Schnittbilddiagnostik mit CT oder MRI sind mit erheblichem logistischen und personellen Aufwand verbunden. Mit einem Navigations- system wird die chirurgische Aktion, d.h. die Instrumentenposition im Patienten, in Bilddaten dargestellt. $\mathrm{Zu}$ unterscheiden sind aktive und passive Navigationssysteme. Aktive Navigationssysteme - Roboter - führen eine vorgeplante chirurgische Aktion selbständig, ohne direkten Eingriff des Operateurs aus. Diese Navigationssysteme spielen in der Wirbelsäulenchirurgie derzeit nur im experimentellen Stadium eine Rolle. Bei passiven Navigationssystemen führt der Operateur das Instrument frei in der Hand und wird in Echtzeit über eine Verknüpfung der Lageinformation des Patienten im Raum, der Position des geführten Instrumentes und der vorhandenen Bildinformation über die exakte Lage des chirurgischen Instrumentes im Patienten informiert. Zusätzlich spürt der Operateur den Wiederstand des Gewebes beim Führen der Instrumente wie bei der konventionellen Technik. Die optische Information wird also mit der taktilen Information verknüpft. Das Prinzip und die klinische Anwendung eines passiven Navigationssystems in der Wirbelsäulenchirurgie sollen im Folgenden dargestellt werden.
Zu unterscheiden sind aktive und passive Navigationssysteme. Aktive Navigationssysteme - Roboter - führen eine vorgeplante chirurgische Aktion selbständig, ohne direkten Eingriff des Operateurs aus. Bei passiven Navigationssystemen führt der Operateur das Instrument frei in der Hand, die optische Information wird also mit der taktilen Information verknüpft.

OP-JOURNAL 2001; 17: 185-190

(C) Georg Thieme Verlag Stuttgart . New York

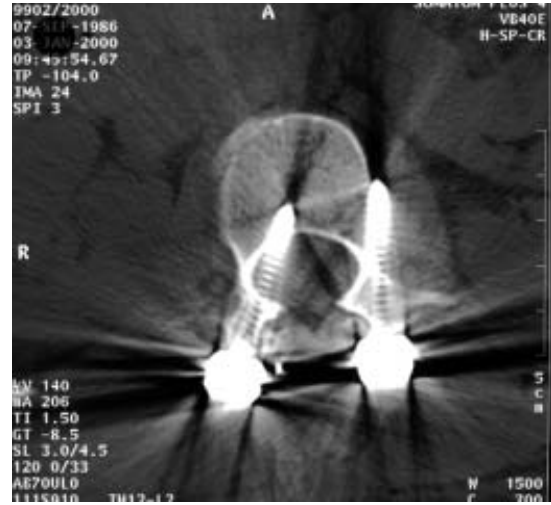

Abb.1 Fehlplatzierte Pedikelschrauben.

$[2,5,6]$ beschrieben. Gerade an der Wirbelsäule kann eine Fehlplatzierung von Implantaten zu nicht unerheblichen Komplikationen führen. Eine nicht korrekte Verankerung der Schraube im Pedikel führt nicht nur zu einem Verlust von Stabilität, sondern kann auch zu Verletzungen der Nervenwurzel, des Rückenmarks oder auch von Blutgefäßen führen.

Die konventionelle Technik verlangt daher vom Operateur eine große Erfahrung, da die Positionierung der Schraube ohne direkte visuelle Kontrolle erfolgen muss. Das Ziel eines Navigationssystems ist es daher, zum einen die Präzision der Schraubenpositionierung zu verbessern, zum anderen soll die Anzahl intraoperativer Fehlbohrungen und Korrekturen reduziert werden. Nicht zuletzt soll die bei Wirbelsäulenoperationen in der Regel hohe intraoperative Strahlenbelastung für den Patienten, aber auch für das medizinische Personal, reduziert werden.

\section{System}

Das Navigationssystem besteht aus einem Hochleistungsrechner, der in der Lage ist dreidimensionale Bilddaten in Echtzeit zu verarbeiten. Angekoppelt an diesen Rechner ist bei den meisten Navigationssystemen eine hochpräzise Infra- bei traumatischen Instabilitäten Wirbelsäule über Pedikelschrauben. Die exakte Positionierung dieser Schrauber tion. In der Literatur wird in der konve Bildverstärker unterstützter Schraubenpositionierung, eine relativ hohe Rate an Fehlplatzierungen von bis $\mathrm{zu} 40 \%$ 

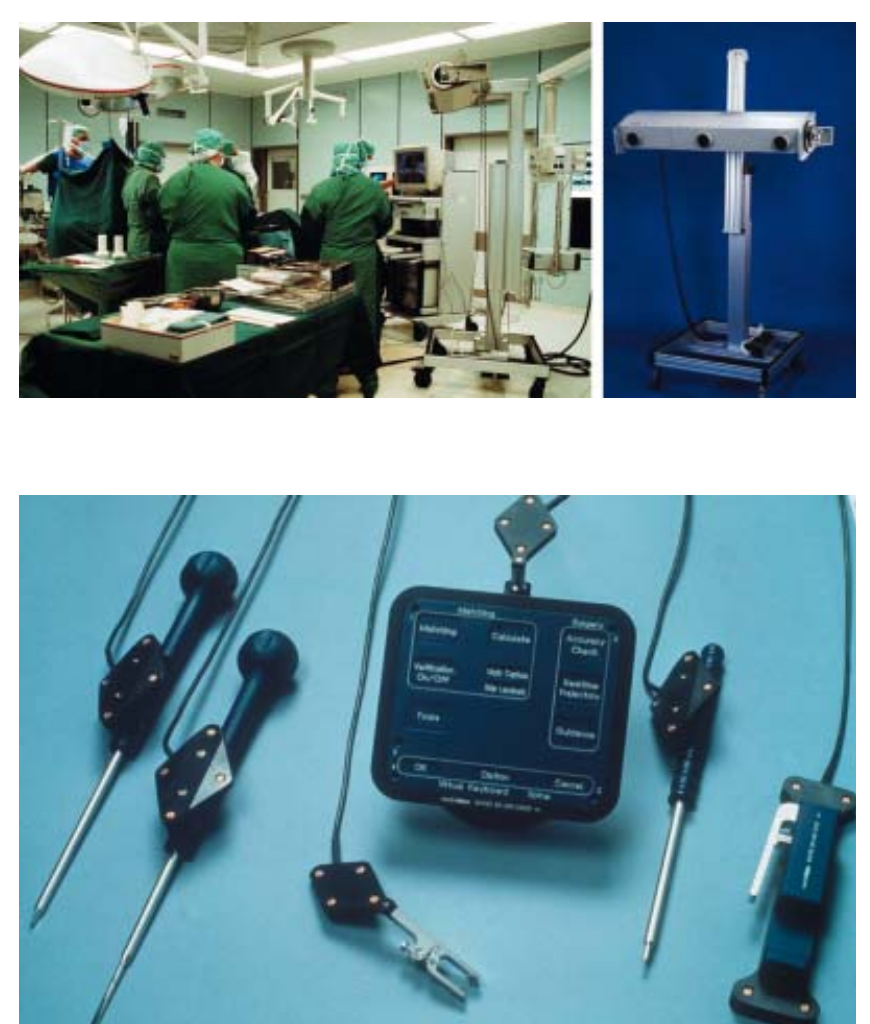

Abb. 3 Navigierte Wirbelsäuleninstrumente.

Abb. 2 Infrarotkamera und Aufbau im OP.

rotkamera. Diese Infrarotkamera ist in der Lage, Instrumente, die mit LED (Light emitting diodes) Markern ausgerüstet sind und entsprechende Infrarotimpulse aussenden, hochpräzise, das heißt mit einer Genauigkeit von ca. $0,3 \mathrm{~mm}$, im Raum zu verfolgen [1].

Die Präzision der Navigation ist entscheidend abhängig von der Kamerapräzision und der exakten Kalibrierung der chirurgischen, navigierten Instrumente.

Im Rechner werden nun die Informationen aus der Instrumentenposition mit den zuvor in den Rechner geladenen Bilddaten, entweder aus einem Schnittbilddatensatz (in der Regel aus der Computertomographie) oder aus Röntgenbildwandlerdaten, die mit einem speziellen kalibrierten und ebenfalls navigierten Röntgenbildverstärker aufgenommen worden sind, verknüpft. Um die Relativbewegungen des zu operierenden Knochens gegenüber der Instrumentenposition auszugleichen, ist es erforderlich, dass diese Relativbewegungen ebenfalls von der Kamera erfasst werden. Daher muss eine so genannte dynamische Referenzbasis (DRB) jeweils an den zu operierenden Knochen angebracht werden.
Die DRB (Dynamische Referenzbasis) erfasst Bewegungen des gerade operierten Wirbelkörpers. Durch Rechenprozesse werden so die Relativbewegungen zwischen Instrument und Knochen vollständig ausgeglichen.

Diese DRB erfasst alle Bewegungen des gerade operierten Wirbelkörpers. Durch Rechenprozesse werden so die Relativbewegungen zwischen Instrument und Knochen vollständig ausgeglichen. Die intraoperative Bedienung des Systems erfolgt unter sterilen Bedingungen durch den Operateur über ein so genanntes virtuelles Keyboard. Das Funktionsprinzip des Keyboards besteht in einer Erkennung der Relativposition eines Zeigeinstrumentes, eines so genannten Pointers, gegenüber einer mit LED-Markern versehenen Metallplatte im Raum

Durch dieses System können alle Standardschritte der Computerbedienung durch den Operateur selbst ausgeführt werden, eine zusätzliche unsterile Bedienung des Systems wird dadurch überflüssig. Wie bereits erwähnt, werden navigierte Pedikelschraubenplatzierungen an der Wirbelsäule entweder CT-basiert oder durch die so genannte C-Arm-Navigation vorgenommen.

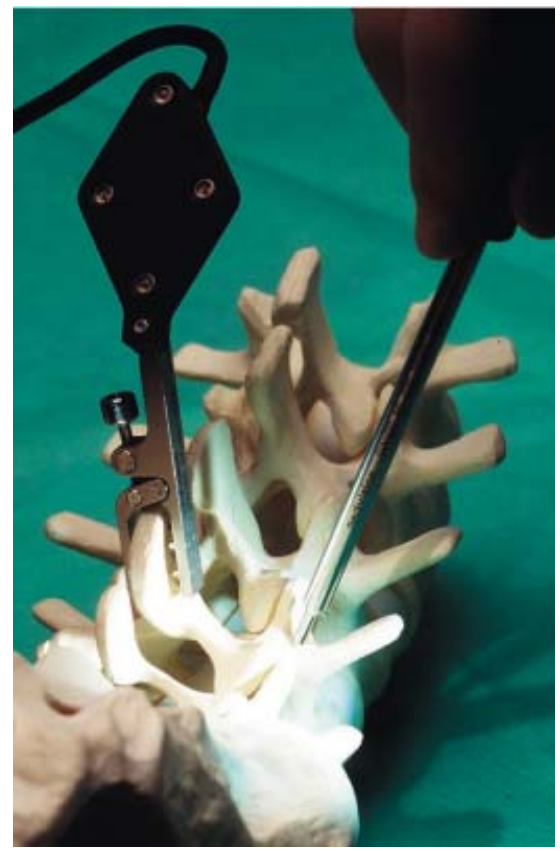

Abb. 4 Dynamische Referenzbasis.

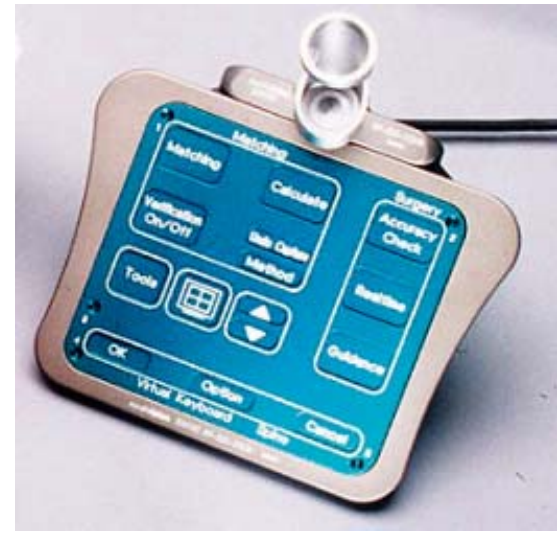

Abb. 5 Virtuelles Keyboard.

\section{CT-basierte Navigation}

Präoperativ ist es erforderlich, bei dem Patienten eine in einem speziellen Modus durchgeführte Spiralcomputertomographie des betroffenen Wirbelsäulenabschnittes durchzuführen. Es ist hierbei darauf zu achten, dass der Wirbelkörper, der instrumentiert werden soll, vollständig erfasst wird. Es ist nun erforderlich, diesen Datensatz in einer präoperativen Planung für die Navigation vorzubereiten und intraoperativ einen Abgleich zwischen der so genannten virtuellen Welt, dem CT-Datensatz und der realen Welt, dem Knochen des Patienten durchzuführen. Hierzu ist die Registrierung von anatomischen Punkten auf den Knochenstrukturen, das so genannte Matching, des Patienten erforderlich. 

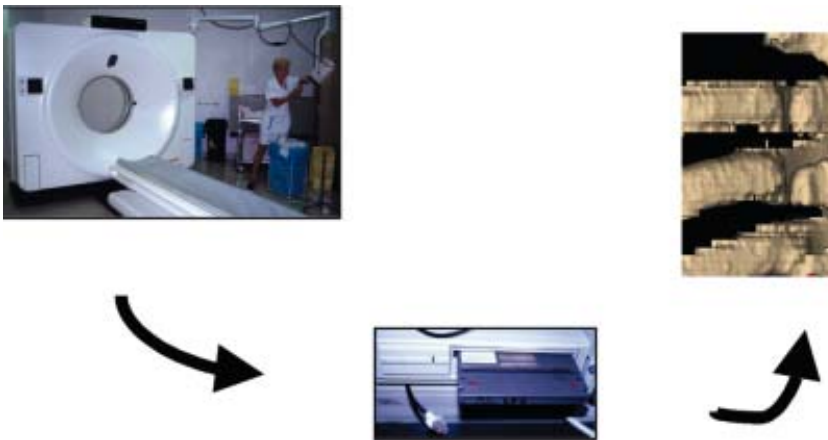

Abb. 6 CT, Datentransfer zur Workstation, dreidimensionale Bildrekonstruktion.

Nach Erstellen der Computertomographie in dem speziellen Modus erfolgt zunächst der Transfer dieser Daten in eine Planungsstation. Der Datentransfer erfolgt entweder über ein Netzwerk, wie in unserer Klinik, oder mobile Datenträger.

Der Operateur muss nun diesen CT-Datensatz für die Navigation vorbereiten. Zunächst wird aus den einzelnen CTSchichten eine dreidimensionale Rekonstruktion erstellt. Hierbei handelt es sich um eine Oberflächenrekonstruktion der Knochenstrukturen, die sich an der Knochendichte der Knochenoberfläche orientiert. Nach erfolgter 3D-Rekonstruktion und Optimierung des Datensatzes durch Herauslöschen von Artefakten werden in dem erstellten dreidimensionalen Datensatz bestimmte Landmarken, die intraoperativ markant auffindbar sein sollten, definiert. Die Qualität dieser Landmarkendefinition ist entscheidend für die Qualität und somit die Präzision der intraoperativen Navigation. Die Landmarken müssen möglichst exakt definiert werden und sollten intraoperativ markant zu erkennen sein. In diesen Datensatz kann auch die angestrebte ideale Position der Pedikelschrauben geplant werden.

Die Präzision der CT-basierten Navigation hängt entscheidend von der Registrierung $\mathrm{ab}$.

Dieser Datensatz wird nun auf den oben genannten Wegen zum OP-System transferiert. Nach dem Starten des Systems, wobei hier auf einen korrekten intraoperativen Aufbau mit einem definierten Abstand zwischen Kamera und Operationsfeld und einer freien optischen Achse zwischen Instrumenten und Kamera zu achten ist, wird der zu instrumentierende Wirbelsäulenabschnitt in herkömmlicher Weise präpariert. An dem Dornfortsatz

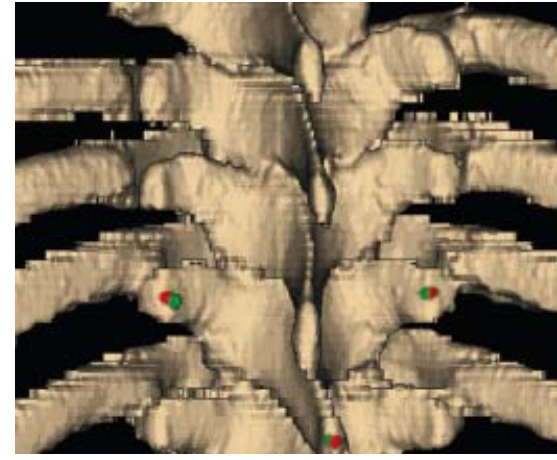

Abb. 8 Paired Point Matching.

des $\mathrm{zu}$ instrumentierenden Wirbels wird die DRB befestigt. Es erfolgt dann die Registrierung des Wirbels, d.h. die virtuelle Anatomie der Daten aus dem CT wird auf die reelle Anatomie übertragen. Zusätzlich zu diesem so genannten pairpoint matching wird in der Regel ein so genanntes surface- oder Oberflächenmatching durchgeführt. D.h. von dem entsprechenden Wirbelkörper werden 12 oder mehr Punkte auf der Knochenoberfläche digitalisiert, wobei sich der Rechner eine Oberflächenstruktur mit möglichst optimaler Übereinstimmung zu dieser Punktwolke auf dem CT-Datensatz sucht. Hierdurch kann in aller Regel die Qualität des Matchings nochmals verbessert werden.

Der für die Navigation absolut entscheidende Schritt erfolgt nun in der Überprüfung der Qualität der Registrierung des zu operierenden Wirbelkörpers (Verifikation) [3]. Der Operateur greift hier-

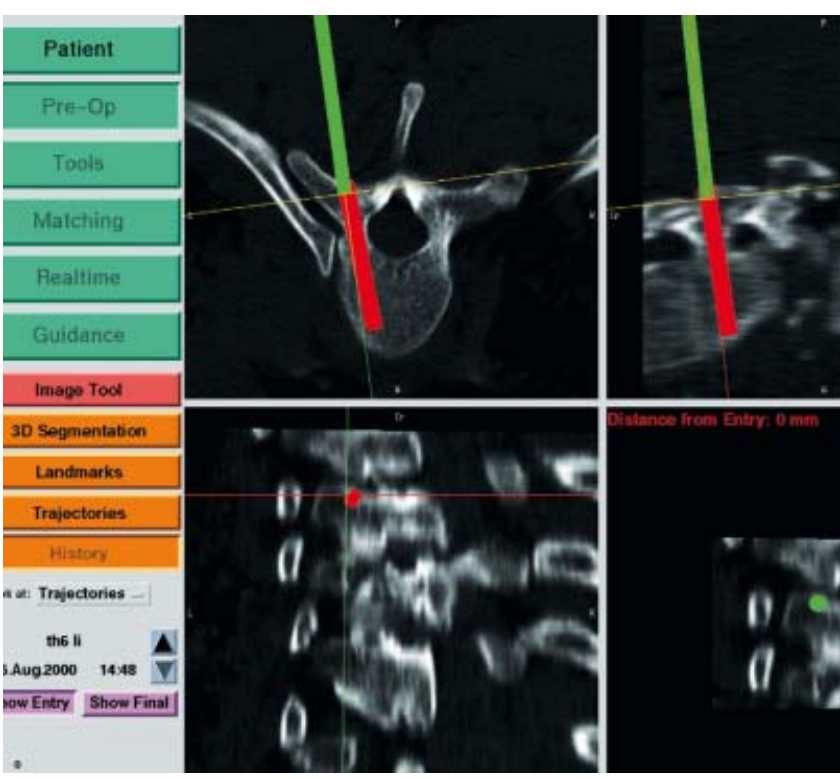

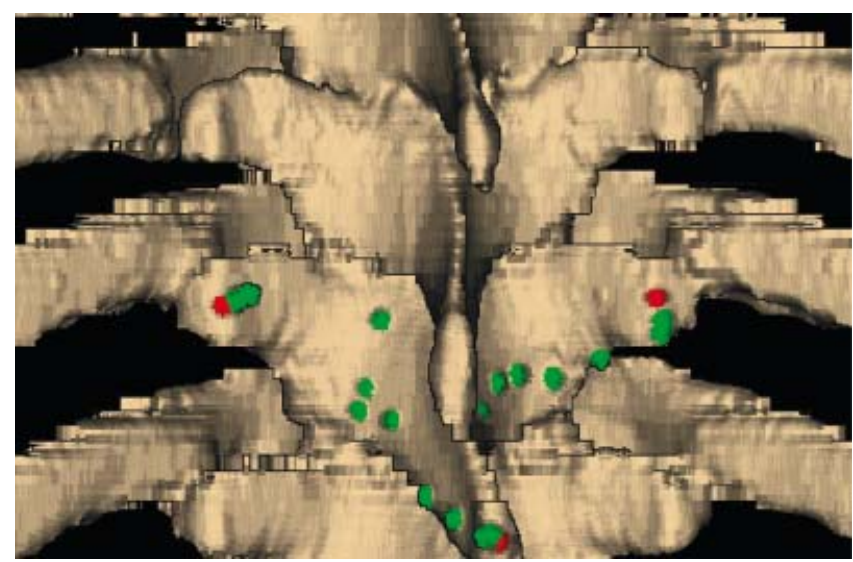

Abb. 9 Surface Matching.

Abb.7 Geplante Position der Pedikelschraube. 
zu beliebige Punkte mit einem navigierten Instrument auf der Wirbelkörperoberfläche $\mathrm{ab}$ und vergleicht diese visuell auf dem Computermonitor bezüglich ihrer Darstellung im System und der realen Anatomie. Nur wenn hier eine exakte Übereinstimmung sowohl bezüglich der Seitverschiebung, der Verschiebung in kraniokaudaler Richtung als auch bezüglich der Tiefe und Rotation angezeigt wird, kann mit ausreichender Sicherheit die Navigation durchgeführt werden.

Probleme beim Matching haben wir bei Osteoporose beobachtet. Hier kann im CT-Datensatz die Knochenoberfläche nicht mit ausreichender Schärfe als dreidimensionale Struktur dargestellt werden. Es kann auch intraoperativ problematisch sein, die präoperativ geplanten Landmarken aufzufinden. Aufgrund der Ähnlichkeit von Wirbelkörpern in bestimmten Wirbelsäulensegmenten, insbesondere im Bereich der Brustwirbelsäule, muss intraoperativ die Höhenlokalisation des referenzierten und instrumentierten Wirbelkörpers mit dem Röntgenbildverstärker in seitlicher Projektion überprüft werden.

Nachdem diese vorbereiteten Schritte durchgeführt wurden, kann die eigentliche Navigation beginnen. Während der Navigation ist auf eine Sichtbarkeit sowohl der dynamischen Referenzbasis als auch der Instrumente für das System zu achten. Die Navigation kann dann anhand einer Darstellung des Wirbelkörpers in 3 zueinander senkrecht stehenden Schnittebenen und zusätzlichen In- formationen bezüglich der Instrumentenposition beim Fortführen der gerade eingeschlagenen Bohrrichtung im so genannten „realtime“-Modus erfolgen. Alternativ kann in einem so genannten „guidance“-Modus die Position des Instrumentes in Relation zur präoperativ geplanten Bohrrichtung im CT-Datensatz dargestellt werden und muss beim Bohrvorgang möglichst in eine exakte Übereinstimmung gebracht werden

Während der Navigation wurden die aktuelle Position des Instrumentes im Wirbelkörper und die Eindringtiefe des Instrumentes in den Wirbelkörper angezeigt. In der CT-Navigation ist es daher möglich nahezu ohne Bildverstärker, nach entsprechender Höhenlokalisation, die Pedikelinstrumentierung durchzuführen. Der Röntgenbildverstärker wird jedoch zum Abschluss der Navigation bei uns zur Positionskontrolle eingesetzt. Aufgrund der möglichen Relativbewegungen der einzelnen Wirbelkörper zueinander muss jeder einzelne Wirbelkörper vor der Instrumentierung getrennt mit der dynamischen Referenzbasis versehen und registriert werden. Repositionsmanöver an der Wirbelsäule werden von dem Navigationssystem nicht erfasst.

\section{BV-Navigation}

Bei der bildwandlerbasierten Navigation erfolgt die Verknüpfung der Instrumentenposition mit Bilddaten aus einem für die Navigation speziell ausgestatteten Röntgenbildverstärker. Hierdurch wird es möglich, die Instrumentenposition in bis zu 4 Bildwandlerebenen gleichzeitig, in einer so genannten virtuellen Fluoroskopie, darzustellen. Diese Darstellung erfolgt, nachdem die Bilder einmal aufgenommen wurden und in das System geladen sind, ohne weitere Durchleuchtungszeit und mit dem großen Vorteil, dass der Röntgenbildverstärker aus dem Operationssitus entfernt werden kann. Zur Navigation wurde ein konventioneller Röntgenbildverstärker mit Infrarotmarken ausgestattet [4].

Die Marker sind starr an dem Röntgenbildverstärker zirkulär befestigt, so dass nahezu jede Position des Bildverstärkers von der Infrarotkamera erkannt werden kann. Da konventionelle BV-Bilder tonnen- und kissenförmig verzerrt sind und in diese verzerrten Bilder keine exakte Projektion eines linearen Instrumentes möglich wäre, ist es erforderlich, diese Röntgenbilder nach einem speziellen Algorithmus zu entzerren. Dieser Entzerrungsalgorithmus muss beim Kalibrieren des BV einmalig für jede einzelne Position festgestellt werden. Um die Entzerrung korrekt zu gewährleisten, ist unmittelbar vor dem Strahlendetektor eine Kalibrierungsplatte mit Markerkreuzen in definierter Größe und definierten Abständen angebracht. Nach der Positionierung und Hochfahren des Systems und dem Anbringen der Referenzbasis an den Dornfortsatz des entsprechenden Wirbelkörpers werden nun BV-Bilder in den entsprechenden Standardprojektionen, a.-p., streng seitlich und in Schrägprojektionen im axialen Verlauf der Pedikelachse aufgenommen und in das Navigationssystem geladen. Nachdem diese Bilder vom Navigationssystem entzerrt

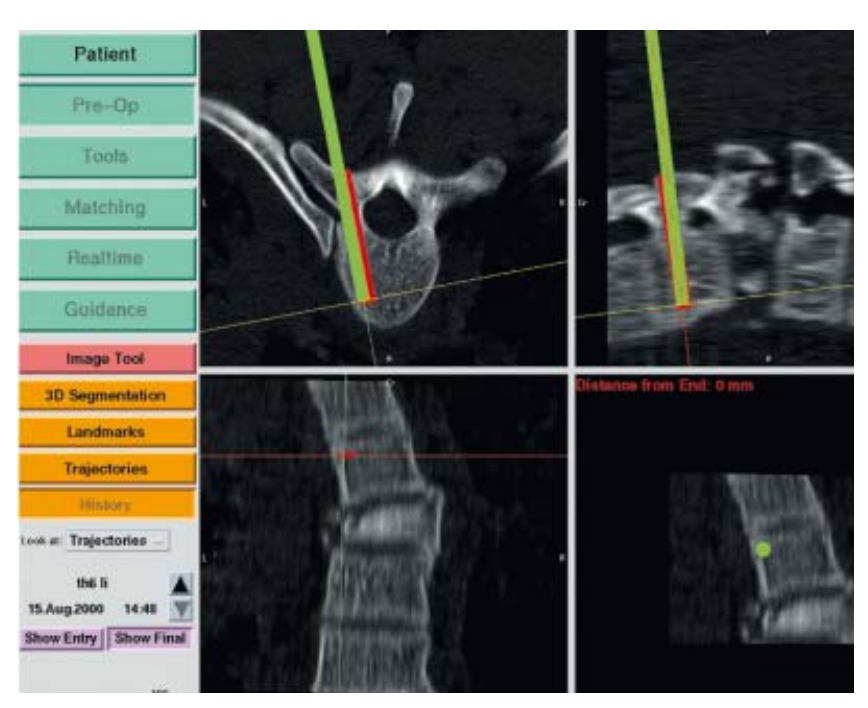

Abb.10 „Guidance“-Modus, Rot: geplante Position, grün: Instrumentenposition.

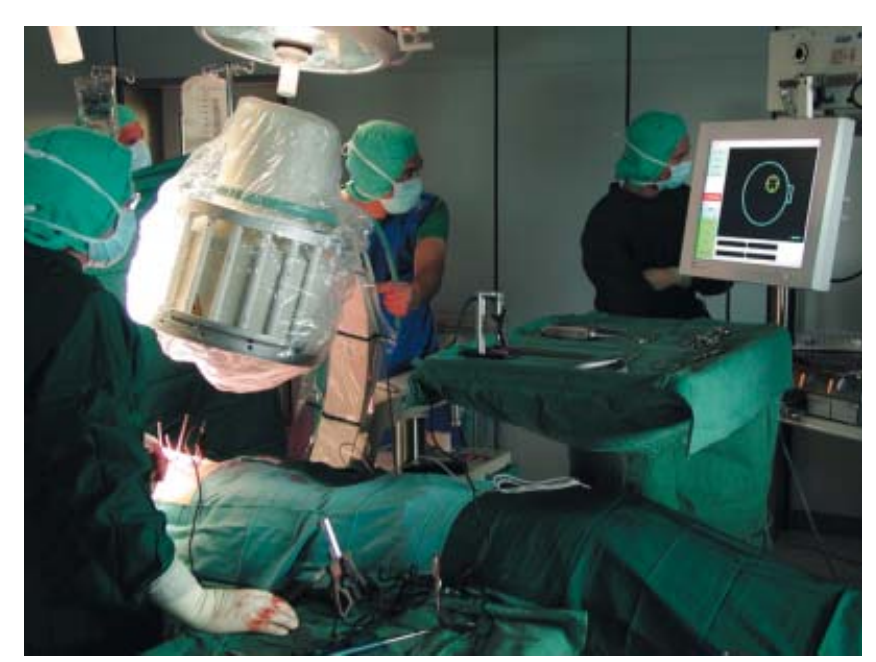

Abb.11 Navigierter BV mit LED Markern-„Alignement“. 
wurden, ist eine weitere Bearbeitung dieser Bilder mit Rotation, Verbesserung des Kontrastes und Vergrößerung durch den Operateur durch das virtuelle Keyboard möglich. Die Markerkreuze, die aufgrund der Kalibrierungsplatte mit abgebildet sind, werden weggerechnet. Das bei der CT-basierten Navigation erforderliche intraoperative Registrieren („matching“) des Wirbelkörpers entfällt hier vollständig. Diese Art der Navigation wird daher auch registrierungsfreie Navigation genannt.

Die BV-Navigation ist registrierungsfrei.

Eine Bearbeitung des präoperativen CTDatensatzes ist daher nicht erforderlich, es muss auch kein für die Navigation speziell geeignetes CT vorliegen. Es ist allerdings streng darauf zu achten, dass in den BV-Bildern, die für die korrekte Schraubenplatzierung erforderlichen Informationen, d.h. die seitliche Abbildung des Wirbelkörpers und die kreis- oder ellipsenförmige Abbildung des Pedikels, das so genannte Auge, im Schrägbild ausreichend gut zu erkennen sind.

Der Nachteil der C-arm-basierten Navigation ist die bei den jetzigen Systemen, durch die Kalibrierungsplatte und die Aufarbeitung der Bilddaten im Navigationssystem noch etwas reduzierte Bildqualität gegenüber konventionellen $\mathrm{BV}$ Bildern und die, wenn auch in verschiedenen Ebenen, nur zweidimensionale Abbildung der knöchernen Strukturen.

Nachdem die Bilddaten aufgenommen und bearbeitet sind, kann nun die Position der entsprechenden Instrumente und Implantate in den verschiedenen Ebenen verfolgt werden, auch ist hier
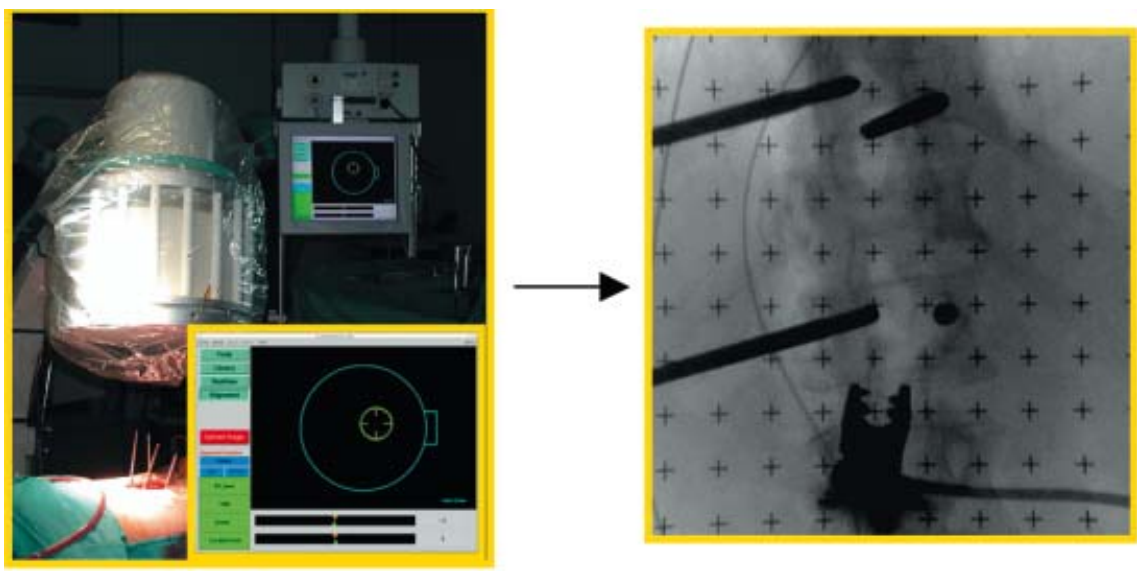

Abb.12 Navigierte Bildwandlerpositionierung.

eine Längenmessung des Bohrvorganges und der Schraubenplatzierung möglich (Abb. 12)

Bei der BV-Navigation werden mehrere Ebenen gleichzeitig dargestellt.

Sowohl die Aufnahme der C-Arm-Bilder als auch die Kontrolle der Implantatpositionierung kann in einem so genannten Alignementmodus kontrolliert werden und somit die intraoperative Strahlenbelastung, aber auch die Zeit bis zur korrekten Positionierung des Röntgenbildverstärkers drastisch reduziert werden. Hierbei wird mit einem Achsinstrument, wie z. B. der navigierten Pedikelahle dem System die gewünschte Durchleuchtungsrichtung des Röntgenbildverstärkers vorgegeben. Der Röntgenbildverstärker wird nun navigiert über eine Zieleinrichtung auf dem Monitor in das Operationsfeld in exakt die gewünschte Positionierung gebracht und somit kann z. B. die Schraubenposition durch ein exakt axiales Rönt-

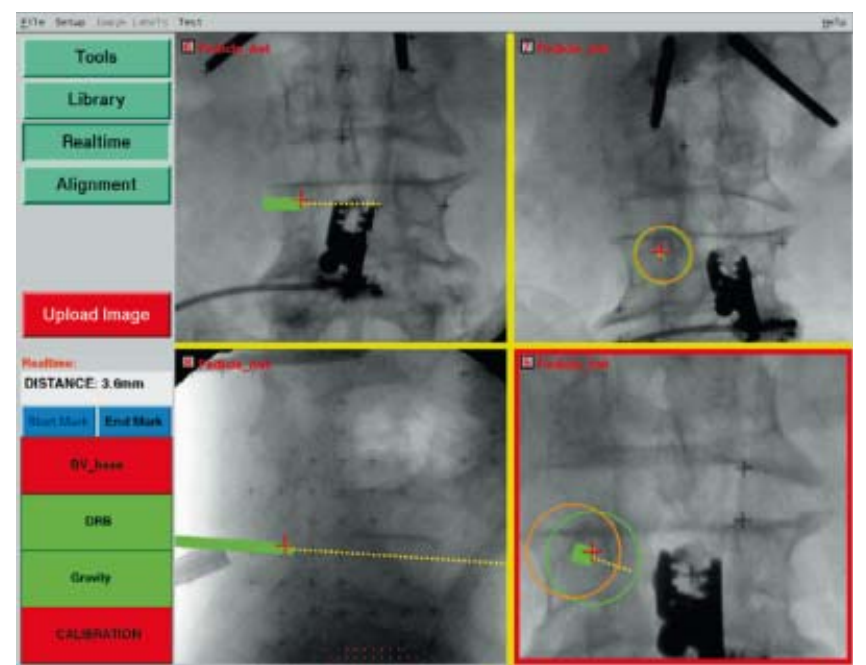

Abb.13 Navigierte Pedikelinstrumentierung. gen der Schraube mit nur einem einzigen BV-Schuss erfolgen.

Vergleicht man CT- und BV-Navigation so liegt der Vorteil der CT-basierten Navigation in der intraoperativen dreidimensionalen Darstellung der knöchernen Strukturen. Gerade bei anatomischen Besonderheiten, wie z.B. Skoliosen oder sehr engen Pedikeln zeigen die dreidimensionale Darstellung und die Möglichkeit der exakten Planung im exakten präoperativen Plan der Verlaufsrichtung des Pedikels deutliche Vorteile (Abb. 10). Die BVbasierte Navigation bietet Vorteile durch die direkt intraoperative Verfügbarkeit ohne zusätzlich präoperativ zu erhebende Datensätze und die Notwendigkeit, einen präoperativen CT-Datensatz zu bearbeiten. Da das Registrierungsverfahren der Wirbelkörper vollständig entfällt, bietet die BV-basierte Navigation gerade an bestimmten Abschnitten der Brustwirbelsäule, an den die Registrierung des CTDatensatzes mit Problemen behaftet ist, Vorteile. Voraussetzung ist jedoch immer eine qualitativ ausreichende Darstellung der anatomischen Strukturen in den Röntgenbildern, wodurch die Anwendbarkeit an den oberen Abschnitten der BWS eingeschränkt sein kann $[7,8]$.

\section{Einsatz bei Notfallindikationen}

Ohne Zweifel bedeutet eine navigierte Pedikelinstrumentierung einen vermehrten apparativen, personellen und abschnittsweise auch zeitlichen Aufwand. Dennoch war es in letzter Zeit zunehmend möglich, mit einer intensiven Schulung des Personals und einer Optimierung der Abläufe, die Vorteile der Navigation mit einer Verbesserung der Präzision und einer Reduktion der Strahlenbelastung, einen Einsatz in der 
Notfallversorgung bei instabilen Wirbelverletzungen mit Verletzungen des Rükkenmarkes einzusetzen. Bei der CT-basierten Navigation wird hierbei die Planung vom Operateur während des Transfers des Patienten aus dem CT in den Operationssaal durchgeführt. Parallel hierzu wird vom geschulten Personal das Navigationssystem intraoperativ vorbereitet und aufgebaut. Der intraoperativ zeitliche Mehraufwand durch Anbringen der Referenzbasis und Registrierung des Wirbelkörpers wird durch die nahezu BVfreie Implantationstechnik der Pedikelschrauben in den meisten Fällen mehr als ausgeglichen. Ähnliches gilt für die C-Arm-basierte Navigation in der Akutversorgung. Auch hier wird der anfängliche Mehraufwand bei der Navigation durch die Reduktion der BV-Zeit und ggf. durch ansonsten notwendige Umpositionierung von Zieldrähten in der Regel mehr als ausgeglichen (8).

Zusammenfassend lässt sich bereits jetzt sagen, dass das derzeit angewendete Navigationssystem einen sehr hohen Standard erreicht hat und in die klinischen Routineabläufe eingegangen ist. Es ist möglich, die Präzision der Implantatplatzierung an der Wirbelsäule und damit die Sicherheit für den Patienten zu verbessern. Dennoch ist die Navigation mit einem hohen Schulungsaufwand verbunden und kann nur durch ständiges Anwenden der Technik optimal genutzt werden. Verbesserungsmöglichkeiten der Systeme liegen in einer Verbesserung der Bildqualität bei C-Arm-basierter Navigation, in einer Verbesserung der Ergonomie und vereinfachter Bedienbarkeit („user interface“), die wir mit Sicherheit bei den Systemen der nächsten Generation bereits finden werden.

\section{Literatur}

1 Nolte L-P, Zamorano L, Visarius H, Berlemann, Langlotz F, Arm E, Schwarzenbach O. Clinical evaluation of a system for precision enhancement in spine surgery. Clin Biomech 1995; 10: 293-303

2 Laine T, Schlenzka D, Mäkitalo K, Tallroth K, Nolte L-P, Visarius H. Improved accuracy of pedicle screw insertion with computer assisted surgery. Spine 1997; 22: 1254-1258

3 Gebhard F, Kinzl L, Arand M. Grenzen der CTbasierten Computernavigation in der Wirbelsäulenchirurgie. Unfallchirurg 2000; 103: $696-701$

4 Hofstetter R, Slomczykowski M, Sati M, Nolte L-P. Fluoroscopy as an imaging means for computer-assisted surgical navigation. Comput-Aided-Surg 4 1999; 2: 65-76

5 Berlemann U. Planning and insertion of pedicle screws with computer assistance. J Spinal Disord 1997; 10: 117-124

6 Schlenzka D, Laine T, Lund T. Computer-assisted spine surgery. Eur Spine J 92000 ; Suppl 1: $57-64$

7 Nolte L-P, Slomczykowski M-A, Berlemann U, Strauss M-J, Hofstetter R, Schlenzka D, Laine T, Lund T. A new approach to compu- ter-aided spine surgery. Fluoroscopy-based surgical navigation. Eur Spine J 9 2000; Suppl 1: 78-88

8 Grützner P-A, Wentzensen A, C-Arm Navigation an der LWS. Osteologie 10 2001; Suppl 2: 86

\section{Dr. med. Paul Alfred Grützner \\ Oberarzt}

Dr. med. Bernd Vock

Oberarzt

Cand. med. Thorsten Köhler

Doktorand

Prof. Dr. med. Andreas Wentzensen

Ärztlicher Direktor

Berufsgenossenschaftliche Unfallklinik

Ludwigshafen

Unfallchirurgische Klinik der

Universität Heidelberg

Ludwig-Guttmann-Str. 13

67071 Ludwigshafen 